University of Wollongong

Research Online

Faculty of Engineering and Information

Faculty of Engineering and Information

Sciences - Papers: Part A

Sciences

$1-1-2016$

Orthogonal experimental design of titanium dioxide - Poly(methyl methacrylate) electrospun nanocomposite membranes for photocatalytic applications

\author{
Andrew Vild \\ University of Wollongong \\ Sara Teixeira \\ Institute for Materials Science and Max Bergmann Centre of Biomaterials \\ Klaus Kuhn \\ Institute for Materials Science and Max Bergmann Centre of Biomaterials \\ Gianaurelio Cuniberti \\ Institute for Materials Science and Max Bergmann Centre of Biomaterials, Dresden Centre for \\ Computational Materials Science (DCCMS), Centre for Advancing Electronics Dresden \\ Vitor Sencadas \\ University of Wollongong, victors@uow.edu.au
}

Follow this and additional works at: https://ro.uow.edu.au/eispapers

Part of the Engineering Commons, and the Science and Technology Studies Commons 


\title{
Orthogonal experimental design of titanium dioxide - Poly(methyl methacrylate) electrospun nanocomposite membranes for photocatalytic applications
}

\begin{abstract}
An orthogonal experimental method was designed to assess the influence of the electrospinning processing parameters on average diameter and distribution of poly (methyl methacrylate) (PMMA) fibers. Based on the orthogonal experimental design analysis, electrospun TiO2-PMMA nanocomposites were processed with the optimal polymer processing conditions to obtain thin fibers with a high overall surface area. Further it was found that the average fiber diameter decreases from $2.0 \pm 0.5$ down to $1.2 \pm 0.2 \mu \mathrm{m}$ with increasing photocatalyst content. Moreover, the wettability of samples was independent of the filler amount, and showed strong hydrophobic behavior. Thermogravimetric analysis showed that for polymer solutions with concentrations higher than $10 \mathrm{wt} \%$, there was a loss of the photocatalytic particles during processing, being more evident for the sample with $40 \mathrm{wt} \%$ particles present in the solution, with a loss of $8 \mathrm{wt} \%$ of ceramic particles. The immobilization of the TiO2 nanoparticles in the polymer fibers led to an increase of the thermal stability. The photocatalytic performance was assessed by using methylene blue (MB). The nanocomposite electrospun fiber membranes had a remarkable photocatalytic activity, especially the one with higher amount of $\mathrm{TiO} 2$, with all the $\mathrm{MB}$ dye being removed from the solution after $100 \mathrm{~min}$, under UV. The orthogonal experimental design is an efficient way to save time and materials in the production of photocatalytic membranes.
\end{abstract}

\section{Keywords}

titanium, design, experimental, orthogonal, poly, dioxide, methyl, methacrylate, applications, photocatalytic, membranes, nanocomposite, electrospun

\section{Disciplines}

Engineering | Science and Technology Studies

\section{Publication Details}

Vild, A., Teixeira, S., Kuhn, K., Cuniberti, G. \& Sencadas, V. (2016). Orthogonal experimental design of titanium dioxide - Poly (methyl methacrylate) electrospun nanocomposite membranes for photocatalytic applications. Journal of Environmental Chemical Engineering, 4 (3), 3151-3158. 


\title{
Orthogonal experimental design of titanium dioxide - poly(methyl methacrylate)
} electrospun nanocomposite membranes for photocatalytic applications

Andrew Vild ${ }^{\mathrm{a}}$, Sara Teixeira ${ }^{\mathrm{b}}$, Klaus Kühn ${ }^{\mathrm{b}}$, Gianaurelio Cuniberti ${ }^{\mathrm{b}, \mathrm{c}, \mathrm{d}}$ and Vitor Sencadas ${ }^{\mathrm{a}}, *$

a School of Mechanical, Materials and Mechatronics Engineering, University of Wollongong, Wollongong, NSW 2522, Australia

b Institute for Materials Science and Max Bergmann Centre of Biomaterials, TU Dresden, 01062 Dresden, Germany

${ }^{c}$ Dresden Centre for Computational Materials Science (DCCMS), TU Dresden, 01062 Dresden, Germany

${ }^{\mathrm{d}}$ Centre for Advancing Electronics Dresden, TU Dresden, 01062 Dresden, Germany

Email: victors@uow.edu.au

Phone: +61 0242214614

\begin{abstract}
An orthogonal experimental method was designed to assess the influence of the electrospinning processing parameters on average diameter and distribution of poly(methyl methacrylate) (PMMA) fibers. Based on the orthogonal experimental design analysis, electrospun $\mathrm{TiO}_{2}$-PMMA nanocomposites were processed with the optimal polymer processing conditions to obtain thin fibers with a high overall surface area. Further it was found that the average fiber diameter decreases from $2.0 \pm 0.5$ down to $1.2 \pm 0.2 \mu \mathrm{m}$ with increasing photocatalyst content. Moreover, the wettability of samples was independent of the filler amount, and showed strong hydrophobic behavior. Thermogravimetric analysis showed that for polymer solutions with concentrations higher than $10 \mathrm{wt} \%$, there was a loss of the photocatalytic particles during processing, being more evident for the sample with $40 \mathrm{wt} \%$ particles present in the solution, with a loss of $8 \mathrm{wt} \%$ of ceramic particles. The immobilization of the $\mathrm{TiO}_{2}$ nanoparticles in the polymer fibers led to an increase of the thermal stability. The photocatalytic performance was assessed by using methylene blue (MB). The
\end{abstract}


nanocomposite electrospun fiber membranes had a remarkable photocatalytic activity, especially the one with higher amount of $\mathrm{TiO}_{2}$, with all the $\mathrm{MB}$ dye being removed from the solution after $100 \mathrm{~min}$, under UV. The orthogonal experimental design is an efficient way to save time and materials in the production of photocatalytic membranes.

\section{Keywords}

Nanocomposite materials; Photocatalytic activity; Surface properties; nanoparticle immobilization

\section{Introduction}

Pharmaceuticals and different micro and nanomaterials, used in daily applications, are disposed of and eventually reach rivers and groundwater. Conventional sewage treatment technologies are ineffective in the removal of such pollutants present at trace levels [1-4], demonstrating an urgent need for innovative technologies that can effectively deal with these compounds [5-7]. Photocatalysis is an alternative since it allows their rapid and efficient removal from water, transforming them into by-products with lower toxicity $[5,6]$. Among several semiconductor materials, titanium dioxide $\left(\mathrm{TiO}_{2}\right)$ has a band gap of $\sim 3.10 \mathrm{eV}[8,9]$, which make it a suitable photocatalyst for the degradation of organic pollutants because of its low cost, unique optical properties, and availability [10].

Catalysts can be employed either in a colloidal or in an immobilized form. The removal of such catalyst nanoparticle following water treatment is a major obstacle towards their applicability in an industrial process. Further, nanoparticle's size, large surface area-tovolume ratio and surface energy, strongly leads to agglomeration during operation, decreasing its efficiency $[11,12]$. For these reasons, immobilized systems are preferable in water treatment, to avoid the costly and additional final filtration process [13-15]. However, the immobilization of photocatalyst nanoparticles to a polymer scaffold is usually weak due to the low attachment of the catalyst onto the supporting material.

Electrospinning is a versatile technique to produce highly porous polymer fibrous membranes with high permeability, small pore size, high specific surface area and good interconnectivity between pores $[16,17]$. Synthetic polymers usually present suitable mechanical properties for handling and separation from reaction media. This process 
can be easily up-scaled for mass production of one-by-one continuous micro and nanofibers from various polymers [16-18].

Electrospinning is a straightforward effective technique to immobilize ceramic particles onto the polymer fibers. Polyamide 6 (PA6) and polyamide 12 (PA12)/titanium dioxide nanocomposite membranes were processed by electrospinning [17, 19]. It was reported that for the same photocatalyst concentration, PA6 solvent casting membranes present enhanced photocatalytic performance when compared to PA6 electrospun nanocomposites. Complete degradation of the methylene blue (MB) dye solution was achieved after $170 \mathrm{~min}$ for the polymer films, while the electrospun membranes only degraded $70 \%$ of the dye, for the same UV radiation exposure time [19]. Electrospun membranes of $\mathrm{TiO}_{2}$-PA12 completely removed the dye after 100 min under UV exposure, and showed suitable thermal and mechanical properties [17].

Poly(methyl methacrylate) (PMMA) is a low cost material that presents good flexibility, mechanical properties, chemical resistance, low density, high durability, and easy availability [20], which makes this polymer a good candidate to use as supporting layer for inorganic nanoparticles, such as $\mathrm{TiO}_{2}$.

Different electrospinning parameters affect fiber formation and ultimately, the average fiber diameter and its distribution. These parameters can be divided in three main categories: a) solution properties (viscosity, solvent properties, and polymer molecular weight; b) jet formation parameters (applied electric filed, flow rate, needle inner diameter, temperature, and moisture). Finally, c) the collection procedure, static or rotating drum or disc collector, and rotation speed determines the fiber diameter and orientation [16]. The number of parameters that influence the fiber diameter and average distribution is thus quite high and experimental design is a powerful tool to determine the parameters with the highest influence on fiber properties.

Orthogonal design is a mathematical method applied to design multilevel experiments, in which selective parameters are chosen to carry out sample experiments from overall experiments on the basis of orthogonality $[21,22]$. This method allows one to evaluate the relative importance of each factor and identifies the ideal values for various factors $[21,23]$. The approach not only saves experimental time to determine the optimum conditions associated to a trial and error approach, but also raw materials.

In the present work, an orthogonal experimental design was used to assess the importance of the individual electrospinning parameters in the formation of the PMMA average fiber diameter and distribution. Based on the performed analysis, the optimum 
electrospinning parameters were chosen to achieve $\mathrm{TiO}_{2}$-PMMA nanocomposite fibers with a smaller average diameter, increasing the area-to-surface ratio of the membranes. Wettability, chemical and thermal properties of the pristine and nanocomposite membranes were characterized. It was found that there is a loss of photocatalytic particles during the electrospinning process, especially for higher filler concentrations. The photocatalytic performance of the pristine and nanocomposite fibers was assessed and correlated to the amount of the photocatalyst present in the fibrous membrane, by following the degradation of MB upon UV radiation exposure and compared to the performance of the $\mathrm{TiO}_{2}$ suspension. Finally, it was found that the photocatalytic performance of the nanocomposite samples is related to the concentration of photocatalyst present in the electrospun fiber matrix and no ceramic filler was lost from the polymeric fibers during the photocatalytic experiments.

\section{Experimental}

\subsection{Materials}

High molecular weight Poly(methyl methacrylate) (PMMA, Elvacite 2041, $M_{w}=$ $450 \mathrm{kDa}$ ) was supplied by Lucite International and titanium dioxide $\left(\mathrm{TiO}_{2} \mathrm{P} 25\right)$ was supplied by Evonik. PMMA was dissolved in a solvent mixture of dichloromethane/dimethylformamide (DCM/DMF, 8/2 vol/vol) at a polymer/solvent ratio of 10/90 (w/w) and stirred at room temperature until complete dissolution. For the nanocomposites solution preparation, the desired amount of $\mathrm{TiO}_{2}$ powder, between 0 and $40 \mathrm{wt} \%$ related to polymer concentration, was added to the DCM/DMF solvent mixture and was sonicated in an ultrasound bath (Soniclean, 250TD) for $4 \mathrm{~h}$. After the $\mathrm{TiO}_{2}$ dispersion, the desired amount of polymer was added to the solution and dissolved with the help of a magnetic stirrer, at room temperature, until complete dissolution.

\subsection{Electrospinning}

The polymer solution was placed in a commercial glass syringe $(10 \mathrm{~mL})$ fitted with a steel needle with different inner diameters $(0.5,1$ and $1.7 \mathrm{~mm})$. Electrospinning was conducted at different applied electric fields (between $0.8 \mathrm{kV} \mathrm{cm}^{-1}$ and $1.4 \mathrm{kV} \mathrm{cm}^{-1}$ ) with a high voltage power supply from Gamma High Voltage. A syringe pump (KDScientific) was used to feed the polymer solution into the needle tip, and the 
electrospun fibers were collected on a grounded collecting plate. The distance between the needle and the collector was kept constant at $15 \mathrm{~cm}$.

\subsection{Experimental design}

Electrospinning processing parameters affecting electrospun fiber diameter and distribution, include molecular weight, moisture, temperature, solution viscosity, applied electric field, among others [24]. An orthogonal design table $L_{9}$ was designed using Orthogonal Design Assistant II software (Sharetop Software Studio). Each orthogonal table has its own mark denoted as $L_{n}(t)^{c}$, where $L$ represent the orthogonal table, $n$ is the total number of experiments, $t$ is the number of levels of each factor, and $c$ is the maximum allowed number of factors. In this work, factors of applied electric field, polymer flow rate, and needle inner diameter, labelled as A, B and C, were investigated (table 1).

Table 1: Factors and levels used for the orthogonal experimental design for PMMA samples.

\begin{tabular}{|c|c|c|c|}
\hline & \multicolumn{3}{|c|}{ Factors } \\
\hline Levels & $\left.\mathbf{A ~ ( E ~ / ~} \mathbf{~ V ~} \mathbf{~ c m}^{-1}\right)$ & $\mathbf{B}\left(\right.$ Flow rate $\left./ \mathbf{~ m L ~ h}^{\mathbf{1}}\right)$ & $\mathbf{C}($ needle diameter $/ \mathbf{~ m m})$ \\
\hline 1 & 0.8 & 1 & 0.5 \\
\hline 2 & 1.0 & 2 & 1 \\
\hline 3 & 1.4 & 8 & 1.7 \\
\hline
\end{tabular}

\subsection{Electrospun fiber membrane characterization}

Electrospun fiber membranes were coated with a thin gold layer using a sputter coater (Polaron, model SC502), and their morphology was analyzed by scanning electron microscopy (SEM) (JCM-6000PLUS Neoscope, from JEOL) with an accelerating voltage of $10 \mathrm{kV}$. The fiber average diameters and their size distribution was calculated over approximately 40 fibers using SEM images at 3000× magnification and Image J software. Contact angle measurements (sessile drop in dynamic mode) were performed at room temperature in a Data Physics OCA20 device using ultrapure water as test liquid. The contact angles were measured by depositing water drops ( $3 \mu \mathrm{L})$ on the sample surface and analyzed with the SCA20 software. Six measurements were 
performed in each sample at different membrane locations, and the average contact angle and standard deviation were taken to characterize each sample.

Infrared measurements (FTIR) were performed at room temperature in an IRAffinity-1S FTIR apparatus from Shimadzu in attenuated total reflectance (ATR) mode from 4000 to $600 \mathrm{~cm}^{-1}$. FTIR spectra were collected after 32 scans with a resolution of $2 \mathrm{~cm}^{-1}$. All measurements were performed at room temperature. The thermal degradation behavior of electrospun samples was characterized by thermogravimetric analysis (TGA) in a Q500 TGA from TA Instruments at heating rate of $20^{\circ} \mathrm{C} \mathrm{min}^{-1}$, under a nitrogen atmosphere.

The degree of porosity of the membrane was determined by the pycnometer method following the procedure described elsewhere [24]. Briefly, the weight of the pycnometer filled with ethanol was measured and labeled as $W_{1}$; the sample with weight $W_{s}$ was immersed in the filled pycnometer. Subsequently, the sample was saturated by ethanol; additional ethanol was added to complete the volume of the pycnometer. Then, the pycnometer was weighted and labelled as $W_{2}$; the sample filled with ethanol was taken out of the pycnometer and the residual weight of the ethanol and the pycnometer was labelled as $W_{3}$. The porosity of the membrane was calculated according to:

$$
\varepsilon=\frac{W_{2}-W_{3}-W_{s}}{W_{1}-W_{3}}
$$

The mean porosity of each membrane was obtained as the average of the values determined in three samples.

\subsection{Photocatalytic activity}

Photocatalytic oxidation of a methylene blue (MB) solution by $\mathrm{TiO}_{2}$-PMMA nanocomposite electrospun fiber membranes was performed under UV irradiation to evaluate the photocatalytic activity of the samples. Tests were performed on $5 \times 5 \mathrm{~cm}^{2}$ electrospun samples (with a thickness of $\sim 70 \mu \mathrm{m}$, measured with a Dualscope MP0R (from Fischer) placed in the inside wall of a borosilicate-glass beaker with MB aqueous solution $\left(50 \mathrm{~mL}, 2 \mathrm{mg} \mathrm{L}^{-1}, \mathrm{pH}=6.8\right)$. Prior to the degradation experiments, the samples were immersed in the $\mathrm{MB}$ solution and stirred for $30 \mathrm{~min}$ in the dark to reach an adsorption-desorption equilibrium of the MB. Subsequently, the samples were exposed 
under continuous stirring to UVA-radiation. The peak wavelength of the lamp was 365 nm (6 Philips 8W mercurial fluorescent lamps - UMEX). The beakers were placed at 15 $\mathrm{cm}$ from the illuminating device. The incident UVA radiation over the sample was measured with a UV34 Lux Meter (PCE) and the average values ranged between 1.6 and $1.7 \mathrm{~mW} \cdot \mathrm{cm}^{-2}$. For analyzing the degradation of $\mathrm{MB}$, the concentration of the $\mathrm{MB}$ was monitored at determined time intervals using a Varian CARY-100 UV-VIS spectrophotometer in the range of 400 to $800 \mathrm{~nm}$. The photodegradation rate of MB was analyzed by monitoring the intensity variation of the main absorption peak ranging from 662 to $665 \mathrm{~nm}$. A control (a fiber membrane without catalyst exposed to UV) and a dark control (a nanocomposite fiber membrane kept in the dark) were also tested.

\section{Results and Discussion}

\section{Optimal electrospinning conditions}

The average fiber diameter and standard deviation (SD) were the evolution indices of the orthogonal experiment for the preparation of the PMMA electrospun membranes. Table 2 presents the results obtained for the different experimental factors investigated, and shows the influence of the interplay of applied electrical field $E$, flow rate $(F)$, and needle inner diameter $(\varnothing)$ on fiber formation. For instance, when the factor is $\mathrm{A}(E)$, the level is $1\left(0.8 \mathrm{kV} \mathrm{cm}^{-1}\right)$, and the evaluation index is the needle inner diameter, then $\mathrm{K}_{1}$ (table 3 ) is mathematically expressed by: $K_{1}=\frac{0.87+0.96+2.36}{3}=1.40 \mu \mathrm{m}$, which represents the mean value of the average diameter for factor $\mathrm{A}$ at level 1 (table 3 ). From the comparison between the different $K$ values (table 3) one can achieve the optimal level of factors for electrospinning experiments of PMMA polymer. Further, the difference between the maximum and the minimum $K$ values are represented by $R$, which denotes the $K$ values range. As an example, the $R$ value for factor $A$ of average diameter is expressed according to: $1.40-1.37=0.03$, and reflects an useful or detrimental effects of the level on the average fiber diameter and its standard deviation. According to this definition, the maximum value of $R$ corresponds to the most important factor $[21,25]$. 
Table 2: Analysis and results of Orthogonal $L_{9}(3)^{4}$ experimental design.

\begin{tabular}{|c|c|c|c|c|}
\hline & \multicolumn{3}{|c|}{ Factors } & \multirow{2}{*}{$\begin{array}{c}\text { Results } \\
\text { Diameter } \pm \text { SD }\end{array}$} \\
\hline Experiment $\mathrm{N}^{\circ}$ & $\mathbf{A}(\mathrm{E})$ & B (F) & $\mathbf{C}(\varnothing)$ & \\
\hline 1 & 1 & 1 & 1 & $0.87 \pm 0.15$ \\
\hline 2 & 1 & 2 & 2 & $0.96 \pm 0.16$ \\
\hline 3 & 1 & 3 & 3 & $2.36 \pm 0.48$ \\
\hline 4 & 2 & 1 & 2 & $0.91 \pm 0.14$ \\
\hline 5 & 2 & 2 & 3 & $1.65 \pm 0.32$ \\
\hline 6 & 2 & 3 & 1 & $1.55 \pm 0.31$ \\
\hline 7 & 3 & 1 & 3 & $1.64 \pm 0.35$ \\
\hline 8 & 3 & 2 & 1 & $0.87 \pm 0.20$ \\
\hline 9 & 3 & 3 & 2 & $1.62 \pm 0.35$ \\
\hline \multicolumn{5}{|c|}{$\begin{array}{l}\text { E - applied electric field } \\
F-\text { solution flow rate } \\
\varnothing-\text { needle inner diameter } \\
\text { SD - means standard deviation }\end{array}$} \\
\hline
\end{tabular}

Fibers with small diameter increase the nanocomposite available surface area, and allows an enhanced exposure of the photocatalyst on the fibers surface [17], consequently, the value of $K$ should be chosen as small as possible. Through the analysis of table 2 and 3, one can observe that the needle inner diameter plays an important role in the fiber average diameter and standard deviation, followed by the flow rate and applied electrical field, respectively. Moreover, the optimal parameters to obtain smooth and thinner fiber average diameter, based on the orthogonal experimental designed performed for the PMMA electrospun membranes is $\mathrm{A} 2\left(1.0 \mathrm{kV} \mathrm{cm}^{-1}\right), \mathrm{B} 1(1$ $\left.\mathrm{mL} \mathrm{h}^{-1}\right)$, and $\mathrm{C} 1(0.5 \mathrm{~mm})$. 
Table 3: Evaluation indices of the orthogonal $L_{9}(3)^{4}$ experiment.

\begin{tabular}{|c|c|c|c|}
\hline & \multicolumn{3}{|c|}{ Factors } \\
\hline Orthogonal indices & $\mathrm{A}(\mathrm{E})$ & B (F) & $\mathrm{C}(\varnothing)$ \\
\hline $\mathrm{K}_{1}$ & $1.40 \pm 0.27$ & $1.14 \pm 0.22$ & $1.10 \pm 0.22$ \\
\hline $\mathrm{K}_{2}$ & $1.37 \pm 0.26$ & $1.74 \pm 0.23$ & $1.17 \pm 0.22$ \\
\hline $\mathrm{K}_{3}$ & $1.38 \pm 0.30$ & $1.84 \pm 0.38$ & $1.89 \pm 0.38$ \\
\hline $\mathrm{R}$ & $0.03 \pm 0.50$ & $0.70 \pm 0.15$ & $0.79 \pm 0.17$ \\
\hline Order of Importance & \multicolumn{3}{|c|}{$C>B>A$} \\
\hline Optimal Level & A2 & B1 & $\mathrm{C} 1$ \\
\hline $\begin{array}{l}K_{i}^{F}=\frac{\sum \text { value of eval }}{R^{F}=\max \left\{K_{l}^{F}\right\}-m} \\
F \text { stands for A, B and } \\
l \text { stands for levels } 1,2\end{array}$ & $\begin{array}{l}\frac{\text { tion indexes c }}{3} \\
\left.K_{l}^{F}\right\} \\
\text { d } 3 .\end{array}$ & me level of $e$ & factor \\
\hline
\end{tabular}

Figure 1 was drawn based on the $K$ values presented in table 3, for the average fiber diameter and standard deviation. It was observed that the applied electrical field does not influence the average fiber diameter, while an increase of polymer average fiber diameter and standard deviation was observed when the flow rate or the needle inner diameter increase, which is in accordance to the expected results. When the flow rate increases, the amount of the polymer solution available at a given voltage is higher, this leads to an increase of the draw volume from the needle tip towards the ground collector, increasing the solvent evaporation time. For this reason a larger polymer crystallization time is needed, and consequently the large fiber average diameter and broader distribution is obtained [16]. When the needle inner diameter increases, the size of the droplet at the needle tip increases, and consequently the surface tension of the droplet decreases, and for the same applied electric field, lower Coulomb forces are required to promote jet initiation. As a result, the acceleration of the jet decreases, and the jet is less stretched during the travel between the needle and the ground collector [16], leading to membranes with higher average fiber diameter and standard deviation, 
as observed in figure 1. From the orthogonal experimental design performed for the PMMA electrospun membranes one can quickly determine the impact of the different factors and their levels, which not only saves experimental time and raw materials, but also provides accurate and reliable optimal electrospun parameters for the PMMA polymer.

Figure 1 - Relationship between the average fiber diameter and the three factors and levels of the orthogonal experimental design, where A is the applied electric field (E), B is the solution flow rate $(\mathrm{F})$, and $\mathrm{C}$ is the needle inner diameter $(\varnothing)$.

Figure 2 shows the morphology of the fiber membranes obtained at different electrical fields (figure 2a-c) and for a higher flow rate (figure 2d). Smooth and beadless fibers were obtained for the different samples. It was also observed that the applied electric field does not influence the average fiber diameter (figure 2a-c). However, when the flow rate increases up to $8 \mathrm{~mL} \mathrm{~h}^{-1}$, a smooth, without the presence of beads and with higher fiber average diameter was observed (figure $2 \mathrm{~d}$ ).

Figure 2 - Poly(methyl methacrylate) electrospun membranes obtained at different processing conditions: a) $E=0.8 \mathrm{kV} \mathrm{cm}^{-1}, \emptyset=0.5$, and flow rate of $1 \mathrm{~mL} \mathrm{~h}^{-1}$; b) $E=1.0 \mathrm{kV} \mathrm{cm}^{-1}, \varnothing=0.5$, and flow rate of $1 \mathrm{~mL} \mathrm{~h}^{-1}$; c) $E=1.4 \mathrm{kV} \mathrm{cm}^{-1}, \emptyset=0.5$, and flow rate of $1 \mathrm{~mL} \mathrm{~h}^{-1}$; and d) $E=1.0 \mathrm{kV} \mathrm{cm}^{-1}, \emptyset=0.5$, and flow rate of $8 \mathrm{~mL} \mathrm{~h}^{-1}$. All experiments were performed at room temperature. Insert is an image of $3 \mu \mathrm{L}$ water droplet, placed on the surface of the membrane, obtained during the WCA measurements.

To process the $\mathrm{TiO}_{2}$-PMMA nanocomposite electrospun membranes, the orthogonal experimental design study performed for the polymer matrix was used to assess the best electrospinning conditions that leads to smooth fibers, without the presence of beads and small average diameter. These fiber characteristics enhance the localization of the photocatalyst ceramic particles onto the fiber surface. $\mathrm{TiO}_{2}-\mathrm{PMMA}$ membranes were electrospun at an electric field of $1.0 \mathrm{kV} . \mathrm{cm}^{-1}$ (A2), a flow rate of $1 \mathrm{~mL} \cdot \mathrm{h}^{-1}$ (B1), and a 
needle inner diameter of $0.5 \mathrm{~mm}(\mathrm{C} 1)$. Figure 3 presents the SEM images of the nanocomposite electrospun membranes obtained for different photocatalyst concentrations (figure 3a-c). Electrospun nanocomposite fibers present a smooth and beadless surface. Although, the photocatalyst nanoparticles do not affect the fibers surface morphology, it was observed that the average fiber diameter increases with the incorporation of $\mathrm{TiO}_{2}$ nanoparticles in the polymer solution (figure 3d). Such behavior is probably due to the fact that the inclusion of the ceramic nanofillers can affect charge density of the solutions. The photocatalyst can be ionized and carry more polymer solution to the needle tip, leading to an increase of the polymer jet between the needle tip and the metallic ground collector, and consequently leads to membranes with higher fiber diameter [16]. It is noteworthy that an increase of the $\mathrm{TiO}_{2}$ nanoparticles concentration present in the solution leads to the formation of clusters on the surface of the polymer fibers, as it can be seen in figure 3 (b and c).

The surface area to volume ratio can be estimated from the average surface diameter $(d)$ [26]:

$$
\text { surface area to volume ratio }=\frac{4}{d}
$$

In the present work it was observed that the surface ar3e to volume ratio decreases from $4.1 \pm 1.7 \mu^{-1}$, calculated for neat PMMA fibers, down to $3.1 \pm 0.7 \mu \mathrm{m}^{-1}$, obtained for the sample with $40 \mathrm{wt} \% \mathrm{TiO}_{2}$.

Figure 3 - Nanocomposite $\mathrm{TiO}_{2}$ - poly(methyl methacrylate) electrospun membranes: a) $10 \mathrm{wt} \% \mathrm{TiO}_{2}$, b) $20 \mathrm{wt} \% \mathrm{TiO}_{2}$, c) $40 \mathrm{wt} \% \mathrm{TiO}_{2}$, and d) evolution of average fiber diameter and size distribution for the electrospun composites processed at the following conditions: $E=1.0 \mathrm{kV} \mathrm{cm}^{-1}$ (A2), flow rate of $1 \mathrm{~mL} \mathrm{~h}^{-1}$ (B1), and $\varnothing=0.5$ (C1). The inserts are images of $3 \mu \mathrm{L}$ water droplet on the surface of the membrane, obtained during the WCA measurements.

Wettability measurements showed that the prepared electrospun membranes have a strong hydrophobic behavior, with an average water contact angle $115 \pm 2^{\circ}$. Further, it was observed that the incorporation of the ceramic photocatalyst does not influence the 
overall pristine and nanocomposite wettability (inserts of figure $2 \mathrm{~b}$ and 3 , and Figure 4). Electrospun pristine and nanocomposite membrane porosity was calculated through equation 2 and it was found that the membrane porosity was in the range $79 \pm 3 \%$, and the immobilization of the ceramic photocatalyst does not influence the overall porosity of the fibers. Similar results were reported for $\mathrm{TiO}_{2}$-poly(amide) electrospun membranes [17].

Figure 4 - Influence of $\mathrm{TiO}_{2}$ concentration on average water contact angle.

Infrared spectroscopy (FTIR) was performed to monitor chemical interactions between the photocatalytic ceramic nanoparticles and the polymer matrix that may occur due to the processing conditions of the pristine PMMA and $\mathrm{TiO}_{2}-$ PMMA electrospun membranes (figure 5). No significant differences were detected in the characteristic FTIR spectra of the samples with different amounts of photocatalyst: no new vibrational modes seem to appear, or are totally suppressed due to variations of the polymer processing conditions (data not shown) or incorporation of the $\mathrm{TiO}_{2}$ nanoparticles in the polymer fiber membrane. The characteristic PMMA absorption bands at 1721, 1449 and $1159 \mathrm{~cm}^{-1}$ are assigned to the $\mathrm{CH}$ stretching, $\mathrm{C}=\mathrm{O}$ stretching, $\mathrm{CH}_{3}$ stretching and $-\mathrm{O}-$ $\mathrm{CH}_{3}$ stretching vibrations, respectively [27].

Figure 5 - FTIR spectra of PMMA electrospun membranes with different amounts of $\mathrm{TiO}_{2}$. Electrospun sample composites processed at the following conditions: $E=$ $1.0 \mathrm{kV} \mathrm{cm}^{-1}$ (A2), flow rate of $1 \mathrm{~mL} \mathrm{~h}^{-1}$ (B1), and $\emptyset=0.5(\mathrm{C} 1)$.

\section{Nanocomposites thermal behavior}

The hydrophobic nature of the PMMA matrix can be confirmed from the TGA and WCA experiments, where no adsorbed water was detected for all the tested samples (figure 6a). Moreover, the thermal stability of the polymer was increased with the incorporation of the ceramic photocatalyst by $\sim 10{ }^{\circ} \mathrm{C}$ when compared to the pristine PMMA electrospun fiber membrane (figure $6 \mathrm{~b}$ ). The onset degradation temperature ( $\left.T_{\text {onset }}\right)$, which can be obtained by extending the pre-degradation portion of the curve to the point of interception with a line drawn as a tangent to the steepest portion of the mass curve occurring degradation, shows a shift towards higher temperatures with the 
incorporation of the photocatalyst (table 4). The midpoint temperature (temperature at $50 \%$ of the loss, $T_{0.5}$ ) is increased compared to the PMMA by more than $20^{\circ} \mathrm{C}$ (figure $6 a)$, in agreement with previous findings reported for the PMMA nanocomposites [2830].

It is interesting to observe that in the case of the $\mathrm{TiO}_{2}$ nanocomposite samples, the degradation step at lower temperatures is reduced with the increasing of the photocatalyst content in the electrospun fiber mats, but it is not totally suppressed (figure 6b). Costache et al. [28] reported that the incorporation of carbon nanotubes (CNTs) into a PMMA film lead to an increase of the thermal stability of the polymer matrix, reducing the lower temperature thermal degradation step.

Figure 6 - a) TGA thermograms of the electrospun nanocomposites, b) DTG plots for the $\mathrm{TiO}_{2}$ - PMMA nanocomposite membranes and, c) Evolution of the amount of photocatalyst present on electrospun nanocomposite membranes.

The residual weight at $500{ }^{\circ} \mathrm{C}$ was recorded for the polymer and nanocomposite samples, and it reveals that there is a loss of photocatalyst nanoparticles weight during the electrospinning process when the $\mathrm{TiO}_{2}$ amount present in the polymeric solution exceeds the $10 \mathrm{wt} \%$ (figure 6c). For the sample with $40 \mathrm{wt} \%$ ceramic powder present in the solution, it was found that the real amount of photocatalyst present in the fibers was $32 \mathrm{wt} \%$, which corresponds to a loss of $8 \mathrm{wt} \%$ of nanoparticles during electrospinning. This loss of $\mathrm{TiO}_{2}$ nanoparticles during the processing is probably due to ionization and acceleration of the ceramic particles between the needle tip and the ground collector, when a high electric filed is applied, and it is in accordance to the average fiber diameter evolution presented in figure 3.

The temperature of the maximum decomposition $\left(T_{d 1}\right.$ and $\left.T_{d 2}\right)$ peak obtained from the DTG curves (figure 6c), showed that no detectable changes were observed for the first degradation step $\left(T_{d 1}\right)$, while a shift toward higher temperature was observed for $T_{d 2}$, with the increasing of the photocatalyst particles present in the fiber membranes (table 4), which shows that the decomposition of the nanocomposite electrospun samples are slower than the pristine polymer membranes. An explanation could be that the higher content of the inorganic filler quenches the applied thermal energy so it doesn't reach the organic PMMA and finally retards the degradation. For that the decomposition is shifted towards higher temperature. 
Table 4: Thermal degradation characteristics of $\mathrm{TiO}_{2}$ - PMMA electrospun samples: $T_{\text {onset }}$, is the onset degradation temperature, $T_{0.5}$, is the midpoint temperature, $T_{d 1}$ and $T_{d 2}$ are the maximum decomposition temperatures of peak 1 and 2 , respectively.

\begin{tabular}{|c|c|c|c|c|}
\hline TiO $_{\mathbf{2}}$ amount & $\boldsymbol{T}_{\text {onset }}$ & $\boldsymbol{T}_{\mathbf{0 . 5}}$ & $\boldsymbol{T}_{\boldsymbol{d 1}}$ & $\boldsymbol{T}_{\boldsymbol{d} \mathbf{2}}$ \\
\hline $\mathbf{w t \%}$ & \multicolumn{5}{|c|}{$\mathbf{}^{\mathbf{C}}$} \\
\hline 0 & 260 & 363 & 298 & 378 \\
\hline 10 & 271 & 382 & 300 & 386 \\
\hline 20 & 284 & 385 & 300 & 390 \\
\hline 40 & 270 & 397 & - & 394 \\
\hline
\end{tabular}

\section{Photocatalytic activity evaluation of $\mathrm{TiO}_{2}-\mathrm{PMMA}$ electrospun membranes}

The photocatalytic activity was tested by degrading MB in an aqueous solution under UV irradiation. To validate the comparisons between the photocatalytic assays, all tested electrospun membranes presented similar thickness $(\sim 70 \mu \mathrm{m})$ and porosity $(79 \pm 3 \%$,), and a PMMA pristine membrane was used as a control. Figure 7a presents the effect of the polymer substrate immersed in the dye solution during UV exposure for $120 \mathrm{~min}$. No significant changes were observed during UV exposition (figure 7a), even for longer period of time (400 min), suggesting that no adsorption occurred due to the strong hydrophobic nature of the polymer matrix (figure 3 and 4 ), MB is stable in UV, and PMMA is inert in the respect to the degradation of MB. For the previous reasons, no degradation occurred. Nevertheless, when the $\mathrm{TiO}_{2}$ particles were immobilized in the fiber surface, decomposition of the dye occurs when the UV source is turned on (figure $7 \mathrm{~b}$ and $\mathrm{c}$ ), suggesting that only the photocatalyst particles present in the polymer membrane are responsible for the MB dye degradation. For the sample with higher amount of $\mathrm{TiO}_{2}(40 \mathrm{wt} \%)$, the absorption band characteristic of the dye $(\sim 665 \mathrm{~nm})$ disappears (figure 7b), and it represents the total discoloration of the dye, leading to a transparent solution.

Figure 7 - Photocatalytic performance of $\mathrm{TiO}_{2}$ - PMMA nanocomposite electrospun membranes under UV: a) UV-VIS spectra of electrospun PMMA (without $\mathrm{TiO}_{2}$ ) sample in a MB solution, collected at different times, b) UV-VIS spectra of electrospun $\mathrm{TiO}_{2}-$ PMMA sample with $40 \mathrm{wt} \%$ of photocatalyst, in a MB solution, collected at different 
times, c) Methylene blue photodegradation performance of the different samples with different $\mathrm{TiO}_{2}$ content and pure $\mathrm{TiO}_{2}$ in suspension (100\%, not immobilized on PMMA), and d) Evolution of the apparent first-order rate constant of the reaction and percentage of degradation after $100 \mathrm{~min}$, for the different processed samples.

To understand the effect on the photocatalytic activity of the different samples, and understand the reaction kinetics, the Langmuir-Hinshelwood pseudo-first order kinetics model was used [31]:

$$
\ln \left(\frac{C}{C_{0}}\right)=-k t
$$

where $C_{0}$ and $C$ represents the concentration of the dye at the reaction time zero and at time $\mathrm{t}$, and $k$ is the apparent reaction rate constant. The $k$ values were calculated via first order linear fit of the data (figure $7 \mathrm{c}$ ). It was observed that the photocatalytic activity of the $\mathrm{TiO}_{2}$ - PMMA electrospun membranes depends on the amount of the immobilized photocatalyst. For comparative reasons, the photocatalytic performance of the $\mathrm{TiO}_{2}$ nanoparticles alone $(100 \%$ in figure $7 \mathrm{c})$ was measured in the same experimental conditions and a value of $0.082 \mathrm{~min}^{-1}$ was calculated for $k$, through equation 3 . The polymer nanocomposite membrane containing the highest amount of ceramic photocatalyst showed the highest $k$.value observed compared to the other composites Nevertheless, the obtained value is smaller than the one observed for the nanoparticles in suspension (figure 7c). The obtained results show that there is a relationship between the amount of the photocatalyst present in the polymer membranes and the apparent reaction rate constant (figure $7 \mathrm{~d}$ ). For instance, the reaction rate constant $k$ for the sample with $40 \mathrm{wt} \%$ of $\mathrm{TiO}_{2}$ is $0.030 \mathrm{~min}^{-1}$ is three times smaller than the one obtained for the ceramic nanoparticles alone, suggesting that the polymer membrane's main function is to support the photocatalyst. A stronger decrease in the reaction rate constant was observed for the $\mathrm{TiO}_{2}$ immobilized in PA12 and PA6 [17, 19]. Despite the reduction of the reaction rate constant, the immobilization of the photocatalyst particles onto the surface of the polymeric fibers seems to be an interesting method for photocatalysis. In a particle suspension, there is a need of an extra step to collect the nanophotocatalyst, which can be time consuming, expensive, and probably giving origin to loss of particles during this process. 
The obtained results suggest that a higher surface area of the polymer fiber membrane leads to a more efficient light interaction with the $\mathrm{TiO}_{2}$ nanoparticles. Moreover, after $100 \mathrm{~min}$, the sample with highest amount of $\mathrm{TiO}_{2}$ nanoparticles achieved nearly $100 \%$ of degradation (figure $7 \mathrm{~d}$ ).

\section{Conclusions}

An orthogonal experimental design was developed to assess the effect of the electrospinning parameters in the PMMA average fiber diameter and distribution. It was observed that while the applied electrical field does not influence the polymer average fiber diameter, increasing flow rate and needle inner diameter leads to fibers with higher average diameter. Based on the analysis of the orthogonal experimental design, electrospun nanocomposite membranes were processed with the experimental parameters that led to the lowest polymer fiber average diameter. Nanocomposite fiber membranes presented an average fiber diameter in between $2.0 \pm 0.5 \mu \mathrm{m}$ down to $1.2 \pm$ $0.2 \mu \mathrm{m}$ for the sample with highest concentration. Moreover, sample's wettability was independent of the filler and showed a strong hydrophobic behavior.

Thermogravimetric analysis showed that for polymer solutions with filler content higher than $10 \mathrm{wt} \%$, there was a loss of the photocatalytic particles during the electrospinning process, and this behavior was more evidenced for the sample with $40 \mathrm{wt} \%$ particles, with a loss of $8 \mathrm{wt} \%$ particles during the process. This is probably due to the interaction between the ionized particles and the applied electric field. The immobilization of the $\mathrm{TiO}_{2}$ nanoparticles in the polymer fibers led to an increase on the thermal stability, almost suppressing the polymer thermal degradation step that occurred at lower temperatures.

The photocatalytic performance of the processed samples was assessed by degrading methylene blue, as a model pollutant. The obtained results showed that the nanocomposite electrospun fiber membranes had a remarkable photocatalytic activity, especially those with a higher amount of $\mathrm{TiO}_{2}$, with nearly all the $\mathrm{MB}$ dye being removed from the solution after $100 \mathrm{~min}$. Finally, the orthogonal experimental design revealed to be an efficient way to save time and raw materials in the production of photocatalytic membranes. Also, the immobilization of the ceramic nanoparticles avoids the extra step usually associated to the recovery of the nanoparticles present in the suspension. 


\section{Acknowledgments}

V. Sencadas thanks the EIS Faculty at UOW for the starting grant. K. Kühn thanks BMBF (Germany) for funding him by the NaViTex project (grant 02WCL1264A). S.

Teixeira thanks the Alfred Kärcher-Förderstiftung.

\section{References}

[1] V.L. Cunningham, S.P. Binks, M.J. Olson, Human health risk assessment from the presence of human pharmaceuticals in the aquatic environment, Regulatory Toxicology and Pharmacology 53(1) (2009) 39-45.

[2] H. Yang, G. Li, T. An, Y. Gao, J. Fu, Photocatalytic degradation kinetics and mechanism of environmental pharmaceuticals in aqueous suspension of TiO2: A case of sulfa drugs, Catalysis Today 153(3-4) (2010) 200-207.

[3] J.H.O.S. Pereira, V.J.P. Vilar, M.T. Borges, O. González, S. Esplugas, R.A.R. Boaventura, Photocatalytic degradation of oxytetracycline using $\mathrm{TiO} 2$ under natural and simulated solar radiation, Solar Energy 85(11) (2011) 2732-2740.

[4] I. Sirés, E. Brillas, Remediation of water pollution caused by pharmaceutical residues based on electrochemical separation and degradation technologies: A review, Environment International 40 (2012) 212-229.

[5] N. Savage, M.S. Diallo, Nanomaterials and Water Purification: Opportunities and Challenges, Journal of Nanoparticle Research 7(4) 331-342.

[6] M. Faramarzpour, M. Vossoughi, M. Borghei, Photocatalytic degradation of furfural by titania nanoparticles in a floating-bed photoreactor, Chemical Engineering Journal 146(1) (2009) 79-85.

[7] K. Kümmerer, The presence of pharmaceuticals in the environment due to human use present knowledge and future challenges, Journal of Environmental Management 90(8) (2009) 2354-2366.

[8] S. Kalathil, M.M. Khan, S.A. Ansari, J. Lee, M.H. Cho, Band gap narrowing of titanium dioxide (TiO2) nanocrystals by electrochemically active biofilms and their visible light activity, Nanoscale 5(14) (2013) 6323-6326.

[9] M.M. Khan, S.A. Ansari, D. Pradhan, M.O. Ansari, D.H. Han, J. Lee, M.H. Cho, Band gap engineered $\mathrm{TiO}_{2}$ nanoparticles for visible light induced photoelectrochemical and photocatalytic studies, Journal of Materials Chemistry A 2(3) (2014) 637-644.

[10] M.L. Yola, T. Eren, N. Atar, A novel efficient photocatalyst based on TiO2 nanoparticles involved boron enrichment waste for photocatalytic degradation of atrazine, Chemical Engineering Journal 250 (2014) 288-294.

[11] S. Anandan, M. Miyauchi, Improved photocatalytic efficiency of a WO3 system by an efficient visible-light induced hole transfer, Chemical Communications 48(36) (2012) 43234325.

[12] D. Kanakaraju, B.D. Glass, M. Oelgemöller, Titanium dioxide photocatalysis for pharmaceutical wastewater treatment, Environmental Chemistry Letters 12(1) (2013) 27-47.

[13] N.J. Peill, M.R. Hoffmann, Chemical and Physical Characterization of a TiO2-Coated Fiber Optic Cable Reactor, Environmental Science \& Technology 30(9) (1996) 2806-2812.

[14] M. Lazar, S. Varghese, S. Nair, Photocatalytic Water Treatment by Titanium Dioxide: Recent Updates, Catalysts 2(4) (2012) 572. 
[15] M. Matsuoka, T. Toyao, Y. Horiuchi, M. Takeuchi, M. Anpo, Wastewater Treatment Using Highly Functional Immobilized TiO2 Thin-Film Photocatalysts, Photocatalysis and Water Purification, Wiley-VCH Verlag GmbH \& Co. KGaA2013, pp. 179-197.

[16] Seeram Ramakrishna, Kazutoshi Fujihara, Wee-Eong Teo, Teik-Cheng Lim, Z. Ma, An introduction to electrospinning and nanofibers, World Scientific2005.

[17] E. Cossich, R. Bergamasco, M.T. Pessoa de Amorim, P.M. Martins, J. Marques, C.J. Tavares, S. Lanceros-Méndez, V. Sencadas, Development of electrospun photocatalytic TiO2-polyamide12 nanocomposites, Materials Chemistry and Physics 164 (2015) 91-97.

[18] A. Krupa, A. Jaworek, S. Sundarrajan, D. Pliszka, S. Ramakrishna, Mechanical Properties an of Electrospun Polymer Fibre-Metal Oxide Nanocomposite Materials, FIBRES \& TEXTILES in Eastern Europe 20(2) (2012) 25 - 27.

[19] M. Lombardi, P. Palmero, M. Sangermano, A. Varesano, Electrospun polyamide-6 membranes containing titanium dioxide as photocatalyst, Polymer International 60(2) (2011) 234-239.

[20] U. Ali, K.J.B.A. Karim, N.A. Buang, A Review of the Properties and Applications of Poly (Methyl Methacrylate) (PMMA), Polymer Reviews 55(4) (2015) 678-705.

[21] G. Xie, Z. Chen, S. Ramakrishna, Y. Liu, Orthogonal design preparation of phenolic fiber by melt electrospinning, Journal of Applied Polymer Science 132(38) (2015) n/a-n/a.

[22] C.-Q. Wang, H. Wang, Y.-N. Liu, Separation of polyethylene terephthalate from municipal waste plastics by froth flotation for recycling industry, Waste Management 35 (2015) 42-47.

[23] M. Liu, Z. Cheng, Y. Jin, X. Ru, D. Ding, J. Li, Optimization and investigation of the governing parameters in electrospinning the home-made poly(I-lactide-co- $\varepsilon$-caprolactone-diOH), Journal of Applied Polymer Science 130(5) (2013) 3600-3610.

[24] C.M. Costa, L.C. Rodrigues, V. Sencadas, M.M. Silva, J.G. Rocha, S. Lanceros-Méndez, Effect of degree of porosity on the properties of poly(vinylidene fluoride-trifluorethylene) for Li-ion battery separators, Journal of Membrane Science 407-408(0) (2012) 193-201.

[25] H.G. Alam, A.Z. Moghaddam, M.R. Omidkhah, The influence of process parameters on desulfurization of Mezino coal by HNO3/HCl leaching, Fuel Processing Technology 90(1) (2009) 1-7.

[26] F. Yang, R. Murugan, S. Ramakrishna, X. Wang, Y.X. Ma, S. Wang, Fabrication of nanostructured porous PLLA scaffold intended for nerve tissue engineering, Biomaterials 25(10) (2004) 1891-1900.

[27] S. Rajendran, T. Uma, Lithium ion conduction in PVC-LiBF4 electrolytes gelled with PMMA, Journal of Power Sources 88(2) (2000) 282-285.

[28] M.C. Costache, D. Wang, M.J. Heidecker, E. Manias, C.A. Wilkie, The thermal degradation of poly(methyl methacrylate) nanocomposites with montmorillonite, layered double hydroxides and carbon nanotubes, Polymers for Advanced Technologies 17(4) (2006) 272-280.

[29] T. Hirata, T. Kashiwagi, J.E. Brown, Thermal and oxidative degradation of poly(methyl methacrylate): weight loss, Macromolecules 18(7) (1985) 1410-1418.

[30] B.J. Holland, J.N. Hay, The kinetics and mechanisms of the thermal degradation of poly(methyl methacrylate) studied by thermal analysis-Fourier transform infrared spectroscopy, Polymer 42(11) (2001) 4825-4835.

[31] J. Yu, G. Wang, B. Cheng, M. Zhou, Effects of hydrothermal temperature and time on the photocatalytic activity and microstructures of bimodal mesoporous $\mathrm{TiO} 2$ powders, Applied Catalysis B: Environmental 69(3-4) (2007) 171-180. 